Revue d'histoire de l'Amérique française

FBS REVUE D.HISTOIRE DE L'AMÉRIQUE FRANÇAISE

\title{
Jean Talon, champion au Canada du gallicanisme royal,
} 1665-1672

D’après sa correspondance avec la Cour de France

Joseph Cossette

Volume 11, numéro 3, décembre 1957

URI : https://id.erudit.org/iderudit/301845ar

DOI : https://doi.org/10.7202/301845ar

Aller au sommaire du numéro

Éditeur(s)

Institut d'histoire de l'Amérique française

ISSN

0035-2357 (imprimé)

1492-1383 (numérique)

Découvrir la revue

\section{Citer cet article}

Cossette, J. (1957). Jean Talon, champion au Canada du gallicanisme royal, 1665-1672 : d'après sa correspondance avec la Cour de France. Revue d'histoire de l'Amérique française, 11(3), 327-352. https://doi.org/10.7202/301845ar d'utilisation que vous pouvez consulter en ligne.

https://apropos.erudit.org/fr/usagers/politique-dutilisation/ 


\section{JEAN TALON, CHAMPION AU CANADA DU GALLICANISME ROYAL, 1665-1672}

\section{D'après sa correspondance avec la Cour de France *}

\section{AVANT-PROPOS ET INTRODUCTION}

L'intendance de Jean Talon a déjà été étudiée dans son ensemble, par Thomas Chapais. Nous n'avons pas la prétention d'ajouter beaucoup de neuf à son étude: nous n'avons pu disposer d'aucun document original dont lui-même n'ait pu se servir. Nous avons simplement voulu réétudier la même question sous le seul aspect religieux, en groupant les faits principaux auxquels l'intendant fut mêlé; souligner de façon spéciale ce qui nous a semblé le trait caractéristique de l'esprit qui présida aux relations entre l'Église du Canada, et l'intendant, entre les années 16651673.

\section{Jean Talon Intendant. Doctrines POLITICO-RELIGIEUSes.}

Le 23 mars 1665, « Louis par la grâce de Dieu, roi de France et de Navarre », signait la Commission établissant Jean Talon son intendant au Canada ${ }^{1}$, et le 27 suivant, un "Mémoire pour lui servir d'instruction »durant son séjour en ce pays. ${ }^{2}$

A ce moment, la charge d'intendant reprenait une grande importance dans le royaume et devenait peu à peu le pivot du système administratif de la monarchie française. Ses attributions sont si vastes: "Police, justice, finances 》, et les termes mêmes en sont si généraux que c'est souvent l'autorité royale dans sa plénitude qu'elle représente. L'intendant doit connaître

* Thèse de licence ès-Lettres-Histoire, Université Laval, Québec. 31) : 3 .

1 Pouvoirs d'intendant pour le Sr Talon, 23 mars 1665, RAPQ (1930-

2 Mémoire du roi pour servir d'instruction à Talon, 27 mars 1665 , RAPQ (1930-31): 5 . 
de toutes les contraventions aux ordonnances, des négligences dans l'administration de la justice, signaler les concussions des magistrats, surveiller la perception des taxes, prévenir et réprimer tout ce qui pourrait menacer l'ordre. ${ }^{3}$ " Désormais, devant l'intendant, tout plie. Nobles, évêques et curés, municipalités et corporations, même pour les affaires les moins importantes, s'adressent à lui. Il devient une Providence ».4 Mais par-dessus tout, l'intendant doit être l'œil du roi omniprésent dans tous les coins du royaume: il doit renseigner le pouvoir royal avec diligence, et faire exécuter les ordres royaux. ${ }^{5}$

A sa nomination comme intendant au Canada, le 23 mars 1665, Jean Talon était bien préparé à remplir cette fonction. En 1653, à l'âge de 28 ans, il avait été commissaire des guerres en Flandres, puis intendant à l'armée de Turenne, où sa diligence à tenir le gouvernement royal au courant de tout lui avait mérité la faveur de Mazarin. Deux ans plus tard, à 30 ans, il reçut l'intendance du Hainaut, territoire important, où Talon manifesta son intelligente activité: il y reçut des marques de l'attention du roi. ${ }^{6}$ C'est de là qu'on vint le prendre en 1665 , pour lui confier l'organisation au Canada de ce que l'on peut appeler une nouvelle province française, avec un gouverneur, un Conseil souverain, un intendant, un vicaire apostolique, qui y remplissait déjà les fonctions épiscopales. ${ }^{?}$

A cette époque, une doctrine politico-religieuse présidait aux relations entre l'Église et l'État en France: le gallicanisme. Ce n'était point de l'anticléricalisme irréligieux ou libre-penseur, au sens moderne du mot, cherchant à saper l'influence du clergé, mais plutôt le souci de maintenir au-dessus de tout la suprématie de l'État: l'Église dans l'État au service de l'État. ${ }^{8}$

3 Halphen et Sagnac, Histoire générale, (Paris, 1944), X: La prépondérance française. Louis XIV: 59-64.

4 Ibid.: $192 \mathrm{~s}$.

5 Loc. cit.

6 Thomas Chapais, Jean Talon, intendant de la Nouvelle-France (Québec, 1904), 17-20.

7 Ibid., passim.

$8 \mathrm{Mgr}$ Fèvre, Histoire de l'Eglise, (Paris, 1885), 37: 433. 
L'origine de cette doctrine est à rechercher dans le haut Moyen âge. Elle dérive de la conception du caractère sacré de la personne royale, qui justifie la tutelle exercée sur l'Église. Formulée sous Philippe Le Bel, depuis longtemps, les rois de France, par un instinct naturel de domination, avaient travaillé à la réaliser. Louis XIV la consacra. ${ }^{9}$ " C'est le système qui, dans l'Église nationale de France, remet au magistrat civil le gouvernement de tout ce qui n'est pas strictement spirituel et la surveillance de toutes les manifestations exérieures de la vie spirituelle ${ }^{10}$ En 1665, la doctrine n'est pas encore formulée aussi strictement, mais nous en trouvons les grandes lignes et l'esprit clairement exprimés dans le « Mémoire du roi pour servir d'instruction à Talon (27 mars 1665) ». Et ce «Mémoire » cadre bien avec les sentiments que le roi exprime dans ses Mémoires (1666), à l'égard des gens d'Église. ${ }^{11}$ Cette doctrine va guider Talon dans l'exercice de sa charge de 1665 à 1672.

La charte de cette doctrine se retrouve dans 2 documents politiques: le premier, la Pragmatique sanction de Charles VII (1438), jamais acceptée par le Pape, mais elle fut mise en pratique par les rois et les parlementaires, et son esprit passa dans les institutions..$^{12}$ Elle soumettait le Pape à l'autorité du concile général, et le liait par les coutumes et les canons de l'Église universelle, ce qui, pratiquement, pour les gallicans, signifiait: l'église gallicane; elle acceptait les disciplines du concile schismatique de Bâle. ${ }^{13}$ Le second document est le Concordat de Bologne, (1516) entre Léon X et François I, imposé à l'occasion de la victoire française sur les troupes pontificales, à Marignan, (15 septembre 1515). » Il met au mains du roi la fortune de l'Église et son haut personnel ».14 Le roi se réserve les nominations aux dignités ecclésiastiques et aux bénéfices, moyennant quelques

9 M. Dubruel, art. «Gallicanisme, » Dict. de théol. cath., (Paris, 1924), VI : col. 1131 .

10 Ibid., col. 1127.

11 Mémoires de Louis XIV écrits par lui-même (2 vol., Paris, 1806), I : $127-156$.

12 M. Dubruel, art. «Gallicanisme», Dict. de théol. cath., col. 1135.

13 Loc. cit.

14 Loc. cit. 
garanties d'honnêteté des candidats; le Pape leur donne les pouvoirs spirituels. Sur le terrain judiciaire, l'Église se réservait uniquement les «causes majeures spécifiées comme telles dans les canons ». Le Concordat de Bologne, «qualifié de privilège », ne dérogeant «que par exception strictement interprétée » à la lumière de la Pragmatique sanction, ${ }^{15}$ a réglé officiellement la situation de l'Église de France jusqu'en 1790; il est donc devenu, jusqu'en 1760, l'une des bases du droit public de l'Église au Canada. ${ }^{16}$

Lorsqu'en 1665, le roi nomme Talon intendant au Canada, l'état de l'Église dans la colonie semble en dehors de l'ordre voulu par le gallicanisme royal. ${ }^{17}$ Des plaintes nombreuses arrivent à la Cour, accusant les gens d'Église de prendre une autorité qui dépasse les bornes de leur véritable profession. ${ }^{18}$ On reprochait spécialement à Mgr de Laval d'avoir, à l'instigation des Jésuites, ${ }^{19}$ lancé l'excommunication contre les trafiquants d'eau-devie aux sauvages. Il s'agissait là d'une question mixte intéressant à la fois la morale et le commerce. Au nom des principes gallicans, ces trafiquants déniaient à l'Église le droit de s'occuper de cette question. ${ }^{20}$ Talon reçut la mission d'enquêter, et de rétablir la situation en faveur de l'autorité temporelle. ${ }^{21}$ C'était pratiquement l'instituer le champion du gallicanisme royal au Canada.

Dans tout ce travail nous ne nous occuperons que des relations de l'intendant avec l'Église du Canada, d'après sa correspondance avec la Cour de France. Cette correspondance est reproduite dans le Rapport de l'Archiviste de la province de Québec, pour les années 1930-1931. Elle couvre environ 178 pages, dont plus de 130 écrites par Talon lui-même. On y trouve 33 lettres de l'intendant à Colbert et 12 «Observations ou Mémoi-

15 M. Dubruel, art. «Gallicanisme », Dict. de théo. cath., col. 1135.

16 Lionel Groulx, « Le Gallicanisme au Canada », RHAF, I (1947) : 56.

17 Mémoire du roi à Talon, 27 mars 1665 , RAPQ $(1930-31): 5$.

18 Loc. cit.

19 Lettres de Marie de l'Incarnation (à son fils, 10 août 1662), éd. Richaudeau, (2 vol., Tournois, 1876), II : 222.

20 Loc. cit.

21 Mémoire du roi, 27 mars 1665, RAPQ (1930-31) : 5 . 
res» sur les affaires du Canada entre les années 1667 et 1673. Le reste de ces écrits se compose d'instructions de la Cour de France, ou des directives annuelles que le ministre Colbert envoie, au nom du roi, à son intendant. Dans toute cette correspondance, on trouve seulement la somme de 4 à 5 pages qui traitent des rapports de l'intendant avec les pouvoirs ecclésiastiques du Canada, y compris le « Mémoire du Roi pour servir d'instruction à Talon 》 (27 mars 1665), et encore, presque toujours ce sont des observations en passant, un mot, une phrase pour rendre compte des affaires ecclésiastiques, au milieu d'autres projets d'ordre économique, industriel, commercial, militaire, passages brefs, mais combien révélateurs de l'esprit qui présidait aux relations entre l'Église et l'État à cette époque.

\section{CHAPITRE PREMIER}

\section{Le MÉmoIre DU ROI (1665): TALON ENQUÊTEUR DU ROI.}

Avant de quitter la France, Talon eut plusieurs entrevues avec Louis XIV et Colbert. Puis, le roi lui fit remettre un long Mémoire pour lui servir d'instruction. Dès le début, avant de parler du danger iroquois, de la misère économique, des grandes œuvres à entreprendre, pour organiser et peupler le pays, le Roi Très Chrétien se préoccupe des affaires religieuses. Il presse Talon de s'enquérir des agissements des Jésuites, des abus de la puissance spirituelle, et de remettre les choses en ordre. A cause des griefs qu'il formule, des principes gallicans qu'il énonce, il vaut la peine de citer ce texte: il servira à Talon de règle de conduite durant toute son administration:

«... ledit sieur Talon sera informé que ceux qui ont fait des relations les plus fidèles et les plus désintéressées dudit pays ont toujours dit que les Jésuites (dont la piété et le zèle ont beaucoup contribué à y attirer les peuples qui y sont à présent) y ont pris une autorité qui passe au delà des bornes de leur véritable profession qui ne doit regarder que les consciences. Pour s'y maintenir, ils ont été bien aises de nommer le Sr évêque de Pétrée pour y faire les fonctions épiscopales, comme étant dans leur 
entière dépendance, et même jusques icy, ou ils ont nommé les gouverneurs pour le Roy en ce pays là, ou ils se sont servis de tous moyens possibles pour faire révoquer ceux qui avaient été choisis pour cet employ sans leur participation, en sorte que, comme il est absolument nécessaire de tenir dans une juste balance l'autorité temporelle qui réside en la personne du Roy et en ceux qui le représentent, et la Spirituelle qui réside en la personne dudit Sr Evêque et des Jésuites, de manière toutefois que celle cy soit inférieure à l'autre, la première chose que ledit Sr Talon devra bien observer, et dont il est bon qu'il ayt en partant d'icy des notions presque entières, est de connaître parfaitement l'estat auquel sont maintenant ces deux authorités dans le pays, et celuy auquel elles doivent estre naturellement. Pour y parvenir, il faudra qu'il voye ici (à Paris, avant son départ pour le Canada) les Pères Jésuites qui ont été audit pays et qui en ont toute la correspondance, ensemble le procureur-général et le Sr Villeray, qui sont les deux principaux du Conseil Souverain estably à Québec, que l'on dit estre entièrement dévoués ausdits Jésuites, desquels il tirera ce qu'ils en peuvent scavoir, sans néanmoins se découvrir de ses intentions $\gg .^{22}$

Portant la signature du roi, ces griefs et l'affirmation de ces principes prennent une importance considérable! En effet, il est intéressant de constater que le roi ne s'arrêtait qu'à trois chefs d'accusation, contrariant plus spécialement les prétentions d'hégémonie du pouvoir royal sur l'Église: les Jésuites auraient empiété sur le pouvoir temporel; ils auraient nommé $\mathrm{Mgr}$ de Laval, parce que «sous leur entière dépendance»; ils auraient fait et défait les gouverneurs, à leur guise. C'était bien suffisant pour lui permettre un rappel des principes de la foi gallicane; «tenir en une juste balance l'autorité temporelle ... et la spirituelle..., de manière toutefois que celle-ci (la spirituelle) fût inférieure à l'autre $» .^{23}$ Le roi aurait pu ajouter encore une longue liste de griefs, contre les Jésuites en particulier que l'on disait les instigateurs de la mesure de Mgr de Laval, contre les ven-

22 Mémoire du roi à Talon, 27 mars 1665 , RAPQ (1930-31) : 5 .

23 Loc. cit. 
deurs d'eau-de-vie aux indiens. On se plaignait de la sévérité intolérable, de l'oppression du clergé; on prétendait que l'autorité religieuse s'aventurait sur le terrain de l'autorité civile, que l'on contraignait les consciences. ${ }^{24}$ Des libelles diffamatoires circulaient de main en main, et s'étaient rendus jusqu'au roi; ils avaient impressionné vivement son entourage. ${ }^{25}$ Pour une bonne part, ils avaient dû inspirer le Mémoire du 27 mars.

A peine arrivé à Québec, le 12 septembre $1665,{ }^{26}$ Talon part sans doute en quête d'informations sur ce premier point si important de son Mémoire, car il n'avait pas beaucoup de temps pour formuler une réponse adéquate: les bateaux pouvaient repartir d'un jour à l'autre. Nous ne savons pas de quelle manière il poursuivit son enquête; mais notons que dès le lendemain de son arrivée il visite les religieuses de l'Hôtel-Dieu, peut-être dans le but de prendre des informations sur ce sujet, car il se présente «sans dire qui il était». Mais la petite sœur Marie de la Nativité «qui avait beaucoup de discernement ${ }^{27}$ perça vite son jeu, au grand amusement de Talon d'ailleurs. En tout cas, dès le 4 octobre, au début d'une longue lettre de 7 pages adressée à Colbert, et traitant de divers sujets, il est en mesure de donner sa réponse. Cette réponse s'efforce d'être objective, mesurée:

«Je dis que le peu de temps qu'il y a que je suis en Canada ne m'a pas encore donné une parfaite connaissance de la conduite Que y ont tenu par le passé les Jésuites qui y sont établis, que cependant ayant fort observé s'ils se servent présentement de l'authorité spirituelle pour affaiblir la temporelle, qui ne doit résider qu'ez personnes qui représentent celle du Roy, j'ay reconnü qu'ils se renferment assez dans l'Etendue de leur véritable profession et jusques icy, il ne m'a paru qu'ils s'empressent pour les affaires du gouvernement, si leur conduite future confirme ou destruict la créance que la présente m'a fait naistre,

24 C. de Rochemonteix, Les Jésuites et la N.-F. au XVIIe siècle (3 vol., Paris, 1895), II : 179; III : 82.

25 Lettres de Marie de l'Incarnation, 30 août 1665, II : 305s.

26 Journal des Jésuites (Québec, 1871), 334.

27 Annales de l'Hôtel-Dieu de Québec (Québec, 1939), 146s. 
j'auray l'honneur d'en advertir le Roy, mais il y a lieu d'Espérer qu'ils ne seconderont pas moins les intentions de sa Majesté dans l'Etablissement du pays qu'ils les font valloir dans l'avancement de la Gloire de Dieu ». ${ }^{28}$

La première impression de l'intendant ne fut donc pas défavorable aux Jésuites, même s'il se réserve pour l'avenir. Depuis l'arrivée de Mgr de Laval, les Jésuites ne faisaient plus partie du Conseil de la colonie, et le P. Ragueneau, qui semble s'être «trop mêlé des affaires publiques et des intérêts privés des colons », avait dû rentrer en France. ${ }^{29}$ Pourtant l'Intendant demeura aux aguets, toujours enclin, il semble, à trouver les Jésuites et le clergé en défaut, à écouter des plaintes contre eux: le résultat de son enquête ne lui semblait peut-être pas vraisemblable, tellement on lui avait annoncé autre chose. Ainsi, en mars 1666, le gouverneur revint de son expédition malheureuse chez les Agniers. Il accusa les Jésuites d'avoir été la cause de son échec, en retenant au fort Chambly les sauvages qui allaient au secours du gouverneur. C'était absurde; mais, note le Journal des Jésuites: Ce qu'il dit «la dessus (selon que nous l'avons apris de $\mathrm{Mr}$ d'Auteuil) fit grande impression sur l'esprit du dernier » (l'intendant). ${ }^{30}$ C'était quelques semaines seulement après l'affaire de la seigneurie de N.-D. des Anges. La résistance des Jésuites à cette spoliation l'avait visiblement irrité. Il faut parler de cette affaire, première manifestation extérieure de ses principes gallicans depuis son arrivée à Québec.

L'instruction de 1665 enjoignait à Talon de faire préparer des habitations pour y recevoir chaque année un certain nombre de colons; il fallait les grouper près des établissements existants. $^{31}$ Pour réaliser ce plan, Talon résolut de former trois bourgs, aux portes mêmes de Québec. Il jeta les yeux sur une terre déjà concédée: la seigneurie de N.-D. des Anges. ${ }^{32}$ Les Jésuites l'avaient obtenue dès 1626 ; la possession leur en avait

28 Talon à Colbert, 4 octobre 1665, RAPQ (1930-31): 32.

29 Rochemonteix, Les Jésuites et la Nouvelle-France. II: 184, note.

30 Journal des Jésuites, 17 mars 1666, 342.

31 Mémoire du roi pour servir d'instruction à Talon, 27 mars 1665, RAPQ (1930-31): 8s.

32 Talon à Colbert, 13 nov. 1666, RAPQ (1930-31): 56. 
été confirmée en $1646^{33}$ et en plusieurs autres circonstances; ainsi le Journal des Jésuites note, "le 6 août 1647: fut mis en possession le fermier de Notre-Dame des Anges $\gg .^{34}$ Les titres de possession étaient sûrs; les Pères avaient rempli convenablement leurs obligations de mettre leur seigneurie en valeur. Le défrichement était sérieusement commencé: environ une centaine d'habitants y étaient déjà installés, et des dispositions étaient prises pour augmenter cette mise en valeur. ${ }^{35}$ Cependant seulement une partie minime de cette terre devait être défrichée, car elle mesurait une lieue de front par 4 de profondeur, et les guerres iroquoises, et le manque de colons, n'avaient certes pas permis là plus qu'ailleurs d'étendre beaucoup le défrichement; mais les Pères avaient fait de grosses dépenses, et voilà qu'au moment où cette terre allait permettre au collège de survivre, le projet de l'Intendant morcelait et amoindrissait considérablement cette seigneurie. ${ }^{36}$

Par ailleurs, Talon estimait que pour accomplir ses instructions ${ }^{37}$ il devait rapprocher et réunir le plus possible les habitations des colons, ${ }^{38}$ et le roi lui avait suggéré de prendre, si nécessaire, les terres non encore mises en culture, même si déjà concédées. $^{39 a}$ Or, il se trouvait que les Jésuites possédaient aux portes de Québec une belle étendue de bonne terre à culture, encore presque entièrement couverte de forêt; y réunir des colons fortifierait les approches de Québec, développerait la colonie tout entière; mais il y avait la question de justice. Est-ce qu'une raison d'utilité, ou de commodité, justifiait l'expropriation de terre, dont la mise en valeur était sérieusement commencée, et dont les titres de possession légitime avaient été plusieurs fois confirmés ? Quand les Jésuites apprirent le projet de Talon, ils réclamèrent leur droit. En guise de réponse, Talon leur envoya un petit billet posant un cas de conscience:

33 Journal des Jésuites, 59.

34 Ibid., 93.

35 Copie de la requête présentée à M. L'intendant par le R.P. Le Mercier, supérieur, touchant nos terres, (le 25 janvier 1666).

36 Loc. cit.

37 Mémoire du Roi à Talon, 27 mars 1665, RAPQ (1930-31) : 8.

38 Ibid.: 56.

39a Ibid.: $8 \mathrm{~s}$. 
«Si un sujet du roi ayant reçu commandement de Sa Majesté de donner toute son application à faire valoir et avancer son service, dans l'établissement d'un pays que Sa Majesté veut procurer, peut en conscience préférer un petit avantage à un beaucoup plus considérable tant au service de Sa Majesté qu'au bien public, et de tout un pays, et par la considération d'un particulier n'embrasser pas le général, surtout en chose notable.

Si même ayant reçu commandement d'établir quarante familles que Sa Majesté veut envoyer au printemps prochain et à cet effet leur préparer des habitations en forme de bourgades toutes cultivées et ensemencées, il peut, par la considération du même intérêt d'un particulier prendre un espace de terre couverte de bois en un lieu où ces pauvres familles seront plus exposées et plus éloignées des secours qui leur seront nécessaires dans les premiers temps, pouvant en prendre une autre couverte aussi de bois, et non en valeur, qui peut non seulement être plus commode aux dites familles mais plus utiles au corps de l'état. ${ }^{39 b}$

En un mot si ce sujet sachant et connaissant parfaitement que ce qu'il fait est beaucoup plus conforme aux intentions qu'il a reçues de ses supérieurs, au service de son roi, et au bien public qu'il doit procurer de toutes ses forces, que tout ce qu'on lui peut proposer ailleurs, peut changer de dessein pour l'intérêt d'un seul ou d'une Communauté ne faisant qu'un membre en l'État»!

En somme, le billet posait le cas suivant: est-ce qu'un serviteur du roi peut, en conscience, préférer un petit avantage à un autre beaucoup plus considérable, pour le service du roi, en considération d'un bien particulier? Beau sujet de bataille à la vérité, et rempli de pièges. Les Jésuites se gardèrent de répondre directement: ${ }^{40}$ ils savaient que le morceau était déjà pris, ${ }^{41}$ car,

39b Les pièces relatives à l'expropriation des terres appartenant aux Jésuites, dont il est question ici, sont conservées aux Archives nationales, Paris, Carton M, 247. Nous les trouvons dans: Appendice, de l'ouvrage de Thomas Chapais, Jean Talon intendant, 490-494. La copie en a été faite pour Chapais par le R.P. de Rochemonteix.

40 Copie de Réponse au dit billet et au dit Cas par manière de requête, dans Chapais, Jean Talon. Appendice, p. 493.

41 Dans une note, (à Québec, octobre 1665) en marge du Mémoire du roi, 27 mars 1665, Talon écrit: «Et desja je commence à faire deffricher pour preparer un lieu ou les nouveaux colons que nous esperons l'année prochaine pourront vivre en communauté ...»RAPQ (1930-31): 16 . 
en décembre, les Jésuites avaient réclamé, et le Journal note:

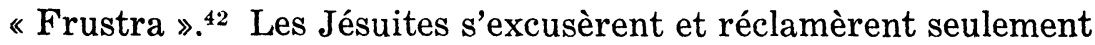
l'attestation écrite que l'intendant avait jugé cette expropriation nécessaire au service et à la satisfaction du roi, afin de pouvoir se justifier devant l'Église et leurs supérieurs de ce désistement de leur propriété. ${ }^{43}$

Au nom de quel principe Talon se croyait-il justifié de s'emparer ainsi d'une propriété privée, sans compensation au propriétaire? - Il semble bien suivre la doctrine des légistes gallicans de l'époque, dont Louis XIV se fait l'écho dans ses Mémoires (1666), quand il écrit au Dauphin: «Tout ce qu'on trouve dans nos États, de quelque nature que ce soit, nous appartient à même titre et nous doit être également cher. Les deniers qui sont dans notre cassette..., ceux qui demeurent dans le commerce de nos peuples... ${ }^{44}$ "Vous devez donc être persuadé que les rois sont seigneurs absolus et ont naturellement la disposition pleine et entière de tous les biens qui sont possédés aussi bien par les gens d'Église que par les séculiers, pour en user en tout temps comme de sages économes, c'est-à-dire suivant le besoin général de l'État $\gg^{45}$ C'était affirmer un principe dangereux, contre le droit naturel de propriété individuelle, et contre le droit de propriété que possède l'Église comme société parfaite.

D'ailleurs, Talon était-il si sûr de lui en dépossédant de leur terre les Jésuites? Quand il fait rapport au ministre sur cette affaire, pourquoi atténue-t-il les termes de son acte? Croyait-il avoir dépassé la portée de ses instructions? Un accommodement avait-il eu lieu entre l'intendant et les Jésuites? Il écrit: « comme j'ai «emprunté » des P. Jésuites et de quelques particuliers le terrain que j'ai fait occuper, on peut leur en laisser la seigneurie et les droits qui seront exigés $\gg .^{46}$ Talon aurait donc été dis-

42 Journal des Jésuites, 26 déc. 1665. Le Journal dit que la réclamation eut lieu le 26 déc. 1665, au sujet des terres de N.-D. du Bon Secours; mais il semble bien qu'il s'agisse là des terres de Notre-Dame des Anges; d'autre part le document cité par Rochemonteix porte la date du 25 janv. 1666.

43 Réponse au billet de Talon. Chapais, Jean Talon, Appendice, 493.

44 Mémoires de Louis XIV, écrits par lui-même, I: $127 \mathrm{~s}$.

45 Ibid., I: 156.

46 Talon à Colbert, 12 nov. 1666, RAPQ (1930-31): 56. 
posé à respecter les droits de seigneurie des Jésuites, et alors leurs objections à cette occupation eussent cessé; mais Colbert en décida autrement. ${ }^{47}$ Les Jésuites se trouvèrent donc totalement dépouillés de leur terre, et perdirent les frais d'exploitation déjà engagés sur cette propriété. Les Jésuites en furent froissés, mais n'osèrent pas pousser plus loin la réclamation de leurs droits. Ils connaissaient sans doute la doctrine alors en vigueur à la Cour, et sentaient qu'ils perdraient leur cause. Mais Talon avait été piqué de cette résistance; il écrit à Colbert: «Je sais qu'on m'assure qu'ils (les Jésuites) en ont mal au cœur. Cependant ils ont la prudence de n'en rien témoigner $\gg^{48}$

\section{CHAPITRE DEUXIÈME}

\section{LE MÉMOIRE DE TALON (1667)}

Jusqu'ici, Talon s'est manifesté le gardien zélé des principes gallicans du roi. Agissait-il comme simple mandataire, ou aussi selon ses convictions personnelles? Il va exprimer ces dernières dans son Mémoire sur l'état présent du Canada (1667). ${ }^{49}$ Une bonne partie du mémoire expose l'état de l'Église du Canada à ce moment.

Talon donne d'abord la description de l'Église: 1 évêque, 9 prêtres, et plusieurs clercs qui vivent en communauté, quand ils sont près de lui dans son séminaire, et séparément, à la campagne, quand ils sont en missions, pour desservir les cures qui ne sont pas encore fondées. Il y a pareillement les Pères de la Compagnie de Jésus, au nombre de 35, la plupart desquels sont employés aux missions étrangères, œuvre digne de leur zèle et de leur piété: "s'il est exempt du mélange de l'intérêt dont on les dit susceptibles, par la traite des pelleteries qu'on assure qu'ils font aux Outaouais et au Cap-de-la-Madeleine: ce que je ne sais pas de science certaine $\gg .{ }^{50}$

47 Colbert à Talon, 5 avril 1667, RAPQ (1930-31): 68 .

48 Talon à Colbert, 27 oct. 1667, RAPQ (1930-31): 79.

49 Mémoire de Talon, 1667, RAPQ (1930-31): 64s.

50 Ibid.:64. 
Talon avait dû prêter l'oreille aux calomnies qui avaient cours contre les Jésuites depuis longtemps. Dès le 3 septembre 1658, le P. de Quen écrivait au R.P. Général: «Improbant in nobis sordidam nescio quam negociationem pellium. Falsum est $\gg^{51}$ Le Général qui n'ignorait pas que les missionnaires ne faisaient aucun commerce, leur recommandait d'en éviter même l'apparence, afin de ne pas donner prise aux calomnies de leurs ennemis. Cette recommandation n'y devait pas mettre fin. Talon signale cette accusation en 1667; Frontenac la renouvelle en 1672, et La Hontan, le grand ami de Frontenac, «qui ne manque jamais l'occasion de manger du Jésuite, - et ce témoignage a son prix, venant d'un tel personnage ${ }^{52}$ dit dans ses Mémoires: «... il n'est pas possible qu'ils fassent le commerce, cela se verrait ».53 En réalité, ce supposé commerce consistait à donner des pelleteries en guise de monnaie, pour se procurer des objets de première nécessité. Le castor était la monnaie d'échange dans les pays d'En-Haut. ${ }^{54}$

Jusqu'ici, encore rien de bien piquant sur la question qui nous occupe; mais Talon continue:

«La vie de ces ecclésiastiques, pour tout ce qui paraît au dehors, est fort réglée, et peut servir de bon exemple et d'un bon modèle aux séculiers qui la peuvent imiter: mais, comme ceux qui composent cette colonie ne sont pas tous d'égale force, ni de vertu pareille, ou n'ont pas tous les mêmes dispositions au bien, quelques-uns tombent aisément dans leur disgrâce pour ne pas se conformer à leur manière de vivre, ne pas suivre tous leurs sentiments, et ne s'abandonner pas à leur conduite qu'ils étendent jusque sur le temporel, empiétant même sur la police extérieure qui regarde le seul magistrat $\gg .^{55}$

Ces accusations sont vagues. Nous aimerions savoir quels gens étaient tombés dans la disgrâce des ecclésiastiques, quand

51 Arch. gén. S.J., cité dans Rochemonteix, Les Jésuites et la N.-F., II : 176 , note 2 .

52 Rochemonteix, Les Jésuites... III : 135, note.

53 La Hontan, Mémoires de l'Amérique septentrionale, (2 vol. Amsterdam, 1705), II : 78.

54 Rochemonteix, Les Jésuites ... III : 139.

55 Mémoire de Talon, $1667, \mathrm{RAPQ}(1930-31): 64$. 
et comment ces derniers avaient empiété sur la police extérieure? Était-ce la question de l'eau-de-vie qui se reposait avec acuité? Peut-être bien! Et nous savons que dans les questions mixtes, touchant à la fois au spirituel et au temporel, la doctrine gallicane réservait au seul magistrat civil le soin de déterminer la conduite à tenir..$^{56}$ Le Mémoire continue: "On a lieu de soupçonner que la pratique dans laquelle ils (les ecclésiastiques) sont... a pour but de partager l'autorité temporelle qui, jusque au temps de l'arrivée des troupes du Roy en Canada, résidait principalement en leurs personnes. A ce mal qui va jusqu'à (gehenner) gêner et contraindre les consciences, et par là dégoûter les colons les plus attachés au pays, on peut donner pour remède l'ordre de balancer avec adresse et modération cette autorité par celle qui réside dans les personnes envoyées par Sa Majesté pour le gouvernement: ce qui a déjà été pratiqué $\gg .57$ Et ainsi, il répétait, la prenant à son compte, une idée déjà exprimée dans le Mémoire du Roi (27 mars 1665). Mais Colbert lui répond que pour le moment la charité royale devra suffire à contenir l'autorité ecclésiastique dans de justes limites. ${ }^{58}$

Enfin, arrive un projet de beaucoup plus de conséquences: l'intendant demande qu'on lui permette « de renvoyer (en France) un ou deux ecclésiastiques de ceux qui reconnaissent moins cette autorité temporelle, et qui troublent le plus par leur conduite le repos de la colonie, et introduire quatre ecclésiastiques entre les séculiers ou les réguliers, les faisant bien autoriser pour l'administration des sacrements, sans qu'ils puissent être inquiétés... » par l'évêque. ${ }^{59}$

Ce que Talon écrit au sujet des consciences gênées et de l'introduction au pays de quatre ecclésiastiques munis de pouvoirs pour administrer les sacrements, «sans qu'ils puissent être inquiétés » par l'évêque, est de nature à nous étonner. Savait-il ce qu'est une juridiction pour administrer les sacrements? Ignorait-il qu'elle ne peut s'obtenir que de l'évêque du diocèse, même

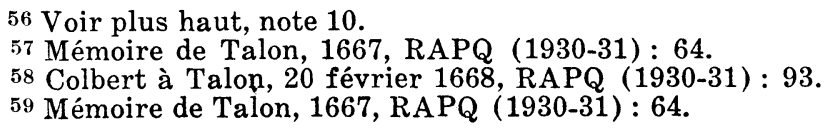


si cet évêque n'a que le titre de vicaire apostolique? ${ }^{60}$ Vouloir amener ici des prêtres pratiquement indépendants de $\mathrm{Mgr}$ de Laval était certes une idée ingénieuse pour atteindre son but: «balancer l'autorité spirituelle et la temporelle», mais de nature à diviser le clergé, à ériger clocher contre clocher. Ce plan de Talon n'était-il pas une ingérence dans la juridiction strictement spirituelle de Mgr de Laval, et cela, au moment où l'intendant dénonçait les soi-disant empiètements de l'Église sur le temporel ? S'il y avait des abus, des sévérités, dans l'administration des sacrements, à qui appartenait-il d'en juger, sinon à l'évêque et à l'Église? Or là était justement le point où les gallicans croyaient de leur droit d'intervenir!

Mais pourquoi tout ce tapage autour des confessionnaux? Quelles consciences souffraient tellement de contrainte? C'est vrai qu'à cette époque la discipline religieuse était sévère, et le pouvoir séculier lui-même punissait rudement les délits contre la morale et les ordonnances ecclésiastiques. ${ }^{61}$ Mais ces sévérités ne semblent pas l'objet des plaintes de Talon; là où les consciences semblaient gênées, c'était dans la traite de l'eau-de-vie aux sauvages, car la peine d'excommunication portée contre eux par Mgr de Laval demeurait toujours en vigueur.

La vente des boissons alcooliques aux sauvages avait été prohibée de temps immémorial dans la colonie. ${ }^{62}$ Le 2 mars 1657 un arrêté du conseil d'État avait ratifié et renouvelé ces défenses sous peine de punition corporelle. ${ }^{63}$ Malgré ces prohibitions, les traitants bravaient les lois et fournissaient aux sauvages, pour

60 Durand de Maillane, dans son Dictionnaire de droit canonique gallican dit, tome $4: 295$, XI, que les prêtres séculiers et les réguliers ne peuvent administrer le sacrement de pénitence sans en avoir obtenu la permission des archevêques ou évêques; puis, page 579 (au mot mission) «... les vicaires apostoliques sont des évêques in partibus infidelium, qui ont une commission particulière du Saint-Siège pour gouverner les églises naissantes dans les pays des infidèles, et qui exercent dans l'étendue de leur vicariat la juridiction épiscopale, tant sur les ecclésiastiques employés à la conversion des infidèles que sur les nouveaux chrétiens ». Talon ne pouvait donc pas amener ici des ecclésiastiques qui administreraient le sacrement de pénitence, sans avoir à se soumettre à la direction de Mgr de Laval.

61 Journal des Jésuites, 106.

62 Arrêts et règlements du Conseil supérieur, et ordonnances et jugements des intendants du Canada (Québec, 1855), 7.

63 Loc. cit. 
avoir à meilleur compte leurs pelleteries, cette eau-de-feu qui les transformait en bêtes féroces; la plus scandaleuse démoralisation en résultait. ${ }^{64}$ En 1660, le mal était devenu si grand que Mgr de Laval crut de son devoir pastoral de prendre une mesure énergique: il lança l'excommunication contre ceux qui faisaient, malgré les ordonnances, la traite de l'eau-de-vie.65 Cet acte épiscopal eût peut-être pu enrayer le fléau, si deux ans plus tard, M. d'Avaugour, n'eût ouvert la porte à de nouveaux excès. ${ }^{66} \mathrm{Mgr}$ de Laval passa en France, obtint le rappel de M. d'Avaugour, et réussit à faire maintenir les défenses antérieures. ${ }^{67}$ Le 6 déc. 1667, à la séance même où le Conseil fut organisé par M. de Tracy, on réitéra la défense de donner ou traiter aux sauvages directement ou indirectement des boissons enivrantes, sous peine d'amende ou de punitions corporelles; cependant, les punitions prévues étaient déjà moins sévères qu'en 1660 , où l'on condamnait à mort pour ce délit. ${ }^{68}$ La défense fut plusieurs fois renouvelée, et Talon y apposait sa signature. ${ }^{69}$ Mais, peut-être devenait-il, de jour en jour, plus sensible aux avantages matériels de la traite et moins touché de ses inconvénients moraux!

En effet, dès le 13 novembre 1666, il écrivait à Colbert: «Quelque temps après mon arrivée icy J'ay proposé de donner des règles de police aux sauvages ... pour former leurs mœurs sur celles des François ... pour avoir droit de les punir quand ils contreviendront aux ordonnances, Les faisant d'ailleurs jouir des avantages qu'ont icy les François, entre autre de l'usage des boissons qui jusques icy leur ont été deffendues, mais j'ai trouvé quelque obstacle que je tâcherai de lever cet hyver $\gg .^{70}$ Quel était cet obstacle? On a lieu de croire que c'était l'attitude de Mgr de Laval et des prêtres, qui maintenaient l'excommunication contre

\footnotetext{
fils), $220 \mathrm{~s}$. II $: 14 \mathrm{~s}$.

65 Mandements... des évêques de Québec (6 vol., Québec, 1887-1890),

66 Journal des Jésuites, 305.

67 Arrêts et règlements du Conseil Supérieur, 7.

68 Journal des Jésuites, 7 oct. $1661,303$.

69 Jugements et délibérations du Conseil Souverain de la N.-F., (6 vol., Québec, 1885-1891), I: $534 \mathrm{~s}$.

70 Talon à Colbert, 13 nov. 1666, RAPQ (1930-31): 58.
} 
la vente de l'eau-de-vie aux sauvages; car depuis lors, Talon se plaint que les consciences sont gênées, contraintes, que l'autorité religieuse empiète sur le domaine temporel, «mesme sur la police extérieure, qui regardent le seul Magistrat $\gg 0^{71}$ Le 29 octobre 1667, il pose nettement la question à Colbert: «Si sa Majesté aprouve ou condamne qu'on traitte des boissons aux sauvages $\gg .^{72}$ Colbert répond que c'est une chose à décider au pays (Canada), "après que l'on aura meurement examiné l'advantage et le desadvantage qui en peuvent arriver...» ${ }^{73}$ Mais entre l'automne de 1666 et la réponse de Colbert (1668), les relations entre l'Église et l'intendant s'étaient tendues. Le 26 août 1667, Talon écrivait: «Je me sens obligé de vous supplier très humblement de suspendre jusqu'à l'arrivée de mon secrétaire vostre créance sur ce qui vous sera avancé (par Tracy) touchant l'église dont l'authorité, bien loing d'estre diminuée, a repris de nouvelles forces et s'est rendue si redoutable que j'ose asseurer que tandis qu'elle demeurera au point où je la vois, vous Monseigneur et ceux qui auront l'honneur de servir icy soubs vos ordres auront beaucoup de peine à faire valloir les bonnes intentions de Sa Majesté pour l'augmentation de cette colonie ... ${ }^{74}$ Le 27 octobre de la même année, il revient encore sur l'idée qu'on portera peutêtre des plaintes contre lui, parce qu'il empêche qu'on ne confonde «la juridiction Magistrale avec l'Ecclésiastique ${ }^{75}$ De toute évidence, quelque chose n'allait plus! Et en effet, il y eut la question de la dîme, qui opposa l'Évêque et Talon; ${ }^{76}$ puis, au mois de mars de 1667, une enquête de Talon sur la conduite de Mgr de Laval, au sujet de la Société de la Ste-Famille, avait été mise en échec, vraisemblablement sous l'influence apaisante de $M$. de Tracy. ${ }^{77}$ Bref, on peut croire que sous l'empire de divers senti$31): 64$.

71 Mémoire de Talon sur l'état présent du Canada, 1667, RAPQ (1930-

72 Talon à Colbert, 29 oct. 1667, RAPQ (1930-31): 88.

73 Colbert à Talon, 20 fév. 1668, RAPQ (1930-31): 96s.

74 Talon à Colbert, 26 août 1667, RAPQ (1930-31) : 76.

75 Talon à Colbert, 27 oct. 1667 , RAPQ $(1930-31): 83$.

76 Abbé Auguste Gosselin, Vie de Mgr de Laval, premier évêque de Québec, (2 vol., Québec, 1890), I: 395-418.

77 Jugements et délibérations du Conseil Souverain de la N.-F., I : 384, et Chapais, Jean Talon, 168-175. 
ments: sympathie pour les marchands, partisans de la traite, désir d'augmenter le commerce, probablement un certain dépit contre l'évêque, dont l'excommunication contre les trafiquants d'eau-de-vie aux sauvages l'offusquait: - car on la considérait comme une entreprise du pouvoir spirituel sur l'autorité civile, ${ }^{78}$ - Talon en vient à considérer comme une erreur les prohibitions portées contre la traite de l'eau-de-vie, erreur préjudiciable au commerce, ou au progrès de la colonie, à l'influence française sur les peuplades indigènes. Aussi, le 10 nov. 1668, sous prétexte spécialement «d'empêcher les désordres » qui naissaient du commerce clandestin de l'eau-de-vie avec les sauvages, et pour introduire ces derniers «dans la société et le commerce des plus honnêtes gens » et par là, les mieux civiliser, il fait lever la prohibition, malgré la peine d'excommunication qui subsistait. Seuls Mgr de Laval et de Tilly, présents à la séance, refusèrent de signer l'arrêt. ${ }^{79}$ Le lendemain, Talon s'embarquait pour la France. Passé dans ces circonstances, cet arrêt ressemble bien «à un coup de tête ${ }^{80}$ de l'intendant contre Mgr de Laval; l'intendant tranche seul une question où la conscience autant que le commerce a son mot à dire; selon les principes du gallicanisme, il donne la priorité à la décision du pouvoir temporel, dans une question mixte.

\section{CHAPITRE TROISIÈME}

\section{SECONDE INTENDANCE: $1669-1673$}

Talon était rentré en France, mais presque aussitôt il reçut une seconde commission pour le Canada. Le 10 mai, le roi la signait, et le 17 suivant, il lui adressait un mémoire succinct de ses intentions. L'intendant recevait instruction de vivre en bonne intelligence avec l'évêque et les Jésuites; de voir à ce qu'ils ne portent pas l'autorité ecclésiastique au delà des bornes qu'elle doit avoir; de travailler à l'établissement des Récollets et de

78 Lettres de Marie de l'Incarnation, 1662, 220s.

79 Jugements et délibérations du Conseil Souverain de la N.F., I: 534s. 80 Lionel Groulx, Histoire du Canada français, (4 vol., Montréal, 19501952), I: 136. 
favoriser les Sulpiciens, "pour modérer la trop grande application des Jésuites à conserver une autorité peut-être trop étendue qu'ils se sont donnée $\gg .81$

C'est pourquoi le 15 mai, le roi envoyait aux Récollets une lettre de cachet, leur enjoignant de s'embarquer sur le premier bateau. ${ }^{82}$ Ils reçurent sans doute cet ordre avec joie. Ils avaient été les premiers missionnaires du Canada, et désiraient toujours y revenir. Mais ils ne savaient peut-être pas dans quel guêpier ils se jetaient, et quel jeu on voulait leur faire jouer! Pour des religieux, être envoyés dans un pays par la puissance séculière, sans être désirés par l'évêque du lieu, ${ }^{83}$ ce n'était certes pas l'idéal. De plus, on les amenait au Canada dans l'intention bien avouée de «balancer les premiers ecclésiastiques, trop bien établis », et pour libérer les consciences de la contrainte qu'elles avaient connue. ${ }^{84}$ Nous avons vu plus haut quelles consciences souffraient de contrainte, et pourquoi. ${ }^{85}$ Le fait d'entrer ainsi dans les vues gallicanes du roi, de Colbert et de Talon, allait leur créer une situation pleine de difficultés. Mais leur arrivée au Canada comblait les vœux de Talon, ${ }^{86}$ et achevait la mise en application de l'instruction royale de 1665: «... Tenir en une juste balance l'autorité temporelle... et la spirituelle...»87 Les Récollets garderaient l'équilibre, en se montrant plus accommodants sur la question de la traite de l'eau-de-vie aux sauvages.

81 Mémoì̃e succinct des principaux poincts des intentions du roy sur le pays de Canada que sa Majcsté veut estre mis ez mains du Sr Talon..., 18 mai 1669, RAPQ (1930-31): 110.

82 Chapais, Jean Talon, 321.

83 Mémoire de Talon sur le Canada au ministre Colbert, 10 nov. 1670, RAPQ (1930-31) : 126.

84 Ibid., 127.

85 Voir plus haut, page 22-25.

86 Mémoire de Talon, 1667, RAPQ (1930-31): 64.

87 « Onze ans après le retour des Récollets au Canada, quelques habitants de Montréal demandèrent à $M$. de Frontenac de leur envoyer des Récollets «pour le repos et le soulagement de leurs consciences ». 《Le procédé avait quelque ressemblance avec celui qui avait amené le retour des Récollets au Canada ». Gosselin, Vie de Mgr de Laval, II : 105. On peut donc conjecturer que les Sulpiciens ne valaient pas mieux que les Jésuites pour calmer certaines consciences. C'est que, même si Mgr de Laval avait retiré l'excommunication contre les trafiquants d'eau-de-vie aux sauvages, il n'en restait pas moins que ces trafiquants n'avaient pas le droit en conscience de saouler les indiens, pour avoir leurs pelleteries. 
Malgré, sans doute, une certaine surprise de les voir arriver sans invitation de l'évêque, les Jésuites saluèrent leur retour avec joie. C'était un précieux renfort apostolique, et la moisson était vaste; les Jésuites pourraient se décharger sur eux du soin des paroisses, comme moins conforme à leur Institut, pour s'adonner tout entier aux missions auprès des sauvages. ${ }^{88} \mathrm{Mgr}$ de Laval aussi les reçut favorablement et leur permit de reprendre leur ancien couvent près de la rivière Saint-Charles. Talon fut-il surpris de cet accueil ? En fit-il part à Colbert ? Ce dernier lui répond qu'il n'a pas douté que l'évêque et les Jésuites ne leur fissent bon accueil. ${ }^{89}$

Le retour des Récollets au Canada fut une réussite du gallicanisme de Talon. Sans doute il était affectionné à cet Ordre, ${ }^{90}$ mais il voyait aussi dans le retour de ces religieux au Canada le moyen de contrebalancer l'influence des Jésuites et de leur enlever le monopole de la direction spirituelle. Il suivait en cela ses impressions, ou plutôt ses préventions, mais il obéissait aussi aux directions de son chef, le ministre Colbert, approuvé par le Roi. ${ }^{91}$

Après son retour en 1670, Talon n'eut pas de démêlés spectaculaires avec l'autorité religieuse; le roi lui avait recommandé dans sa seconde commission (1669) de "vivre en bonne intelligence avec l'évêque et les Pères Jésuites, mais d'observer qu'ils ne portent pas l'autorité ecclésiastique au-delà des bornes qu'elle doit avoir ». Pourtant, il faillit avoir encore une passe d'armes avec Mgr de Laval. Il avait eu vent que les Jésuites de Rome poussaient les affaires pour obtenir l'érection canonique du siège de Québec en évêché. ${ }^{22}$ Un bon gallican ne pouvait tolérer une telle indépendance de conduite de la part du clergé! Il fit connaître à Sa Grandeur qu'il ne devait son titre «que de Sa Majesté, qui seule pouvait le lui faire accorder, et qu'il était de ses intérêts de tenir tous ses établissements d'elle, parce que d'elle seule,

88 Relation des Jésuites de 1670. (3 vol., Québec), 2.

89 Colbert à Talon, 11 fév. 1671, RAPQ (1930-31): 147.

90 Mémoire sur le Canada, 10 nov. 1670, RAPQ (1930-31) : 127.

91 Lettre du Roi à Talon, 15 mai 1669, RAPQ (1930-31): 101s; et Mémoire, 18 mai 1669, RAPQ (1930-31) : 110.

92 Mémoire de Talon sur le Canada, 10 oct. 1670, RAPQ (1930-31) : 127. 
il en dépendait ».93 $\mathrm{Mgr}$ de Laval dut s'incliner. D'ailleurs Talon pouvait s'appuyer ici sur le Concordat de Bologne, qui concédait au roi de France la nomination aux dignités ecclésiastiques. Monseigneur passa donc en France dans l'espoir de hâter le règlement de cette affaire, qui va traîner jusqu'en $1674 .^{94}$ Dès 1668, des bulles avaient été préparées à Rome, plaçant le siège de Québec dans la dépendance directe du Saint-Siège, mais les intrigues $\mathrm{du}$ gallicanisme officiel créaient des difficultés: on voulait Québec dépendant d'un archevêché français; Rome ne voulut pas céder et finalement gagna son point; mais il fallut du temps, et c'était Mgr de Laval qui en souffrait.

Dans ce dernier épisode, nous voyons encore une fois l'intendant se faire le champion zélé du gallicanisme de son maître. Le vicaire apostolique du Canada doit tout attendre du roi, et de lui seul; il est de «ses intérêts de tenir tous ses établissements de lui ». Cette dernière phrase prend le ton d'un ultimatum; il fallait obéir!

\section{CONCLUSION}

Nous finissons cette revue de la carrière du Grand Intendant, dans ses relations avec l'Église canadienne. Il eût été plus agréable sans doute de mettre en relief la grande œuvre économique qu'il a commencée en notre pays: projets, initiatives, travaux, dont la plupart n'eurent guère de suite immédiate. Mais nous avons préféré nous arrêter à l'aspect religieux de son administration, reflet de l'absolutisme royal à l'égard de l'Église de France. Et nous nous sommes arrêté à un seul point de cet aspect: son inspiration gallicane. Par là, nous n'avons pas voulu diminuer la mémoire de Talon, ni ombrer sa réputation; nous avons simplement voulu mieux connaître la source de quelques misères qui ont entravé l'action des hommes d'Église au Canada, à cette époque, misères dues, pour une bonne part, aux doctrines politico-religieuses, dont les gouvernants du XVIIe s. étaient les héritiers, et dont ils se faisaient les défenseurs.

93 Loc. cit.

94 Wilfrid Paradis, «L'érection du diocèse de Québec et l'opposition de l'archevêque de Rouen, 1662-1674 », RHAF, IX: 465-501. 
Nous avons remarqué avec quel soin l'autorité politique s'est appliquée à balancer l'autorité spirituelle, et à la tenir au second rang. Il est vrai qu'au début de la colonie cette dernière exerçait une influence extraordinaire: la colonie n'était pratiquement alors qu'une mission et un comptoir de commerce. Cette situation devait changer avec le temps. Mais cette application du roi et de l'intendant à balancer l'autorité spirituelle à l'avantage de la temporelle, à toujours soupçonner l'Église d'empiéter sur le domaine temporel, est de nature à nous étonner aujourd'hui. Nous regrettons qu'une union plus harmonieuse, un appui réciproque et bienveillant, basés sur l'indépendance des deux autorités, chacune dans leur sphère respective, n'aient pas régné. Mais cette rivalité, cette jalousie de l'autorité politique à l'égard de l'Église était justement le fruit de cette doctrine que l'on a qualifiée du nom de Gallicanisme.

Talon, en bon serviteur du roi, s'en est fait le défenseur zélé, partout où il la crut en danger. Nous ne suspectons pas sa bonne foi personnelle: il s'est montré un bon chrétien pratiquant, zélé pour soulager les pauvres; ${ }^{95}$ heureux de la conversion des protestants; ${ }^{96}$ mais nous regrettons qu'il n'ait pas compris que les avantages commerciaux devaient laisser le pas aux exigences de la morale dans la question de l'eau-de-vie; et que ses idées gallicanes aient fait de lui un champion très zélé du gallicanisme.

JOSEPH COSSETTE, s.j.

95 Annales de l'Hôtel-Dieu de Québec, $169 \mathrm{~s}$.

96 Talon à Colbert, 7 oct. 1665 , RAPQ (1930-31) : 39 . 


\section{BIBLIOGRAPHIE}

\section{1 - Sources imprimées}

Annales de l'Hôtel-Dieu de Québec 1636-1716. Composées par les Révérendes Mères Jeanne-Françoise Juchereau de St-Ignace et Marie Andrée Duplessis de Ste-Hélène Anciennes Religieuses de ce Monastère, Editées dans leur Texte original avec une Introduction et des Notes par Dom Albert Jamet de l'Abbaye de Solesmes. A l'Hôtel-Dieu de Québec, MCMXXXIX. (1939). 447 pp., $32 \mathrm{~cm}$.

Arrêts et règlements du Conseil Supérieur de Québec et ordonnances et jugements des intendants du Canada, imprimés sur une adresse de l'Assemblée législative du Canada. Québec, de la presse à vapeur de E. R. Fréchette, 1855. 650 pp., $25.5 \mathrm{~cm}$.

Charlevoix, le P. de, s.j. Histoire et description générale de la Nouvelle-France avec le Journal historique d'un voyage fait par ordre du Roi dans l'Amérique Septentrionale. A Paris chez la Veuve Ganeau, MDCCXLIV (1744), avec Approbation et Privilège du Roy. Tome second. 501 pp., $17 \mathrm{~cm}$. Histoire anecdotique, qui passe vite sur les difficultés entre le spirituel et le temporel, sans donner les motifs doctrinaux.

Constitutiones Societatis Jesu et Epitome Instituti. Romæ, Apud curiam præpositi generalis, 3a Ed. 1943.

Correspondance échangée entre la Cour de France et l'intendant Talon pendant ses deux administrations dans la NouvelleFrance, (23 mars 1665 - 11 juin 1677), 3-183, dans le Rapport de l'Archiviste de la province de Québec pour 193031. Redempti Paradis, Imprimeur de Sa Majesté le Roi, (Québec) 1931.

DURAND DE MAILlane, Dictionnaire du droit canonique et de pratique bénéficiale conféré avec les maximes et la jurisprudence de France, c'est-à-dire, avec les usages et libertés de l'Eglise gallicane, les Pragmatiques et Concordats, les Ordonnances, Edits et Déclarations de nos Rois, les Arrêts des Parlements et du Grand Conseil. 6 tomes. $20 \mathrm{~cm}$. A Paris chez La Villette, Libraire, rue du Battoir, No 8. (M.DCC. LXXXVII) 1787. Avec approbation du Roi. Code officiel du droit canonique gallican.

Edits, Ordonnances royaux, déclarations et Arrêts du Conseil d'Etat du Roi concernant le Canada. Imprimés sur une adresse de l'Assemblée législative du Canada. Revus et corrigés d'après les Pièces originales déposées aux Archives 
Provinciales, vol. I. Québec: de la presse à vapeur de E. R. Fréchette, 1854.648 pp., $25.5 \mathrm{~cm}$.

Inventaire des documents concernant l'Eglise du Canada sous le régime français, (1610 à 1708), 155-463. Dans le Rapport de l'Archiviste de la province de Québec pour 1939-1940. Secrétariat de la province, Redempti Paradis, Imprimeur de Sa Majesté le Roi, (Québec) 1940.

Journal des Jésuites, publié d'après le manuscrit original conservé aux archives du Séminaire de Québec, par MM. les abbés Laverdière et Casgrain. Québec. Chez Léger Brousseau, Imprimeur-Editeur. 1871.403 pp. $29 \mathrm{~cm}$.

Jugements et délibérations du Conseil Souverain de la NouvelleFrance. Publiés sous les auspices de la Législature de Québec, vol. I. Imprimerie A. Côté et Cie. Québec, 1885. 1084 pp., $26.5 \mathrm{~cm}$.

La Hontan. Mémoires de l'Amérique septentrionale ou la suite des voyages de $M$. le Baron de La Hontan. Tome second, 257 pp. A Amsterdam, chez François l'Honoré et Compagnie, MDCCV.

Lettres de Marie de l'Incarnation, (née Marie Guyard) première supérieure du monastère des Ursulines de Québec, Nouvelle édition augmentée et annotée par l'abbé Richaudeau. Tome second. Vve H. Casterman, éditeur pontifical, Tournai, 1876. $377 \mathrm{pp} ., 22 \mathrm{~cm}$.

Mandements, lettres pastorales et circulaires des Evêques de Québec, publiés par Mgr H. Têtu et l'abbé C. O. Gagnon. Volume premier (1660-1740). Imprimerie générale de A. Côté et Cie. (Québec) 1887. 488 pp., $22 \mathrm{~cm}$.

Mémoires de Louis XIV, écrits par lui-même, composés pour le grand Dauphin, son fils, et adressés à ce prince. Mis en ordre et publiés par J. L. M. de Gain-Montagnac. Première partie. Paris, Garnery, Libraire. M.DCCCVI. Tome 1 et 2, 231 pp. $20 \mathrm{~cm}$.

Relations des Jésuites contenant ce qui s'est passé de plus remarquable dans les missions des Pères de la compagnie de Jésus dans la Nouvelle-France. Ouvrage publié sous les auspices $\mathrm{du}$ gouvernement canadien. Volume III, embrassant les années de 1656 à 1672, et une table analytique des matières contenues dans tout l'ouvrage. Augustin Côté, EditeurImprimeur. Québec, 1858. 688 pp., $25.5 \mathrm{~cm}$.

Rituel du diocèse de Québec publié par l'ordre de Monseigneur l'Evêque de Québec. A Paris chez Simon Langlois, MDCCCIII (1703), avec privilège du Roi. 673 pp., $22 \mathrm{~cm}$. 


\section{2-Etudes}

ARQUILlIÈRE, H. X. Article Gallicanisme, col. 1096-1137, dans: Dictionnaire de Théologie Catholique, contenant l'exposé des doctrines de la Théologie catholique, leurs preuves, et leur histoire, commencé sous la direction de A. Vacant et de E. Mangenot, continué sous celle de E. Amann, avec le concours d'un grand nombre de collaborateurs. Tome sixième, Paris, VI. Librairie Letouzey et Ané. 1924, $27.5 \mathrm{~cm}$. Etude générale de la doctrine catholique, approuvée par l'Eglise.

ChapaIs, Thomas, Jean Talon intendant de la Nouvelle-France (1665-1672). Québec Imprimerie de S. A. Demers, 1904. $540 \mathrm{pp} ., 23 \mathrm{~cm}$. Il donne un bon aperçu des idées et gestes gallicans de l'intendant.

FÈVRe, Mgr, Histoire de l'Eglise, T. 37. Paris. Louis Vives, libraire-éditeur, $1885.668 \mathrm{pp}$., $22 \mathrm{~cm}$. Histoire à tendance polémique.

FLICHE et MARTIN, Histoire de l'Eglise depuis les origines jus$q u$ 'à nos jours. T. 19 , 1ère et 2 ème parties. Les luttes politiques et doctrinales aux $X V I I^{e}$ et $X V I I I^{e}$ siècles, par E. Préclin et E. Jarry. Bloud et Gay, 1956. On y donne un aperçu rapide des affaires religieuses de la Nouvelle-France aux $\mathrm{XVII}^{\mathrm{e}}$ et $\mathrm{XVIII}{ }^{\mathrm{e}}$ siècles et du Gallicanisme en France, vers la même époque.

GaRneaU, F.-X., Histoire du Canada depuis sa découverte jus$q u$ 'à nos jours. Seconde édition corrigée et augmentée. Tome premier. Imprimé par John Lovell, Québec, 1852. 377 pp., $22 \mathrm{~cm}$.

Gosselin, l'abbé Auguste, Vie de Mgr de Laval premier évêque de Québec et apôtre du Canada, 1622-1708. Tome premier. Imprimerie de L. J. Demers et Frère. Québec, 1890. 672 pp., $22 \mathrm{~cm}$.

GoYAU, Georges, Les Origines religieuses du Canada. Une épopée mystique. Bernard Grasset, Editeur. Paris, 1924. 285 pp., $18.5 \mathrm{~cm}$. Il décrit le climat religieux de la Nouvelle-France jusqu'à l'arrivée de Talon au Canada.

GroulX, Chan. Lionel, «Le Gallicanisme au Canada sous Louis XIV », Revue d'Histoire de l'Amérique française. Publiée par l'Institut d'Histoire de l'Amérique française, Montréal, Outremont, Canada. Vol. I, (1947) : 54-90. Il laisse Talon pratiquement dans l'ombre.

GroulX, Chan. Lionel, Histoire du Canada français depuis la découverte. T. I. L'Action Nationale. (Imprimerie popu- 
laire, Limitée, 434 est, rue Notre-Dame, Montréal). 221 pp., $22 \mathrm{~cm}$.

HALPHEN et SAgNAC. La prépondérance française, Louis XIV (1661-1715) par Philippe Sagnac et A. de Saint-Léger dans Peuples et civilisations, Histoire générale. T. X. Presses Universitaires de France. Paris, 1944. 694 pp., $23 \mathrm{~cm}$.

LATREILle, André, L'Eglise catholique et la Révolution francaise. Vol. I, Le Pontificat de Pie VI et la Crise française, (17751799). Librairie Hachette, Paris VIe, 1946. 279 pp., $22 \mathrm{~cm}$. Il décrit l'état du gallicanisme, surtout au XVIII ${ }^{\mathrm{e}}$ siècle.

LECLER, J., Article «Anticléricalisme », dans Catholicisme, Hier, Aujourd'hui, Demain, col. 633-638. Encyclopédie en sept volumes, dirigée par G. Jacquemet du clergé de Paris. Tome Premier. A-Béthel. Letouzey et Ané, Paris. 1948. $28 \mathrm{~cm}$.

LE JEUNE, le R.P. L., O.M.I. Dictionnaire général de biographie, histoire ... du Canada. Université d'Ottawa, Canada (1931). Tome second. 829 pp., $27 \mathrm{~cm}$.

PARADIS, Wilfrid H., ptre. "L'érection du diocèse de Québec et l'opposition de l'Archevêque de Rouen, 1662-1674. » Revue d'Histoire de l'A mérique française. Vol. IX : 465-501. Thèse de doctorat en droit canonique, présentée devant la Faculté de droit canonique de l'Université catholique de Paris.

Rochemonteix, le P. Camille de, s.j. Les Jésuites et la NouvelleFrance au XVII e siècle, d'après beaucoup de documents inédits. Avec portraits et cartes. Tome deuxième, $536 \mathrm{pp}$. et troisième, 694 pp. Letouzey et Ainé, Editeurs. Paris, 1895. $22.5 \mathrm{~cm}$. Il s'attache à défendre avec ardeur les Jésuites, sans mentionner très fort le mot gallicanisme. Cet ouvrage considérable n'a pas de table analytique.

Stanley, G. F. G. « The Indians and the Brandy Trade during the Ancien Regime », Revue d'Histoire d'Amérique française, vol. IV : 489-505, mars 1953. Excellente synthèse de la question. 